\title{
Performances estimation and potential of the Hyper-Kamiokande detector
}

\author{
Stephane Zsoldos* \\ Queen Mary University of London \\ E-mail: s.zsoldos @qmul.ac.uk
}

Hyper-Kamiokande is a next generation underground water Cherenkov detector, based on the highly successful Super-Kamiokande experiment. It will serve as a far detector, $295 \mathrm{~km}$ away, of a long baseline neutrino experiment for the upgraded J-PARC beam. It will also be a detector capable of observing - far beyond the sensitivity of the Super-Kamiokande detector — proton decay, atmospheric neutrinos, and neutrinos from astronomical sources.

The inner segment called the Inner-Detector has a cylindrincal shape of $70.8 \mathrm{~m}$ in diameter and $54.8 \mathrm{~m}$ in height. This main active volume for physics measurments is viewed by an array of 40000 inward-facing 20" photosensors, which provide a photo-cathode coverage of roughly $40 \%$. Hyper-Kamiokande employs a ring-imaging water Cherenkov detector technique and as a consequence, the capability of a water Cherenkov detector largely relies on the performance of its photosensors. The dimension of the photo-sensors and their density are subject to an optimization that takes into account the required signal identification efficiencies, background rejection power, and cost.

In this presentation we will discuss the physics potential of Hyper-Kamiokande with emphasis of non-accelerator physics.

35th International Cosmic Ray Conference - ICRC2017

10-20 July, 2017

Bexco, Busan, Korea

${ }^{*}$ Speaker. 


\section{Introduction}

Hyper-Kamiokande is the succesor of the Super-Kamiokande experiment which was awarded with the Nobel Prize in 2015 for the joint discovery with the SNO experiment of atmospheric neutrino oscillations. Compared to Super-Kamiokande, this new experiment will consists of two cylindrical water tanks that are $60 \mathrm{~m}$ in height and $74 \mathrm{~m}$ in diameter, bringing the total volume up to $0.5 \mathrm{Mm}^{3}$ per tank — see Figure 1 .

Hyper-Kamiokande will be a multipurpose neutrino detector with a rich physics program that aims to address some of the most significant questions facing particle physicists today. Oscillation studies from accelerator, atmospheric and solar neutrinos will refine the neutrino mixing angles and mass squared difference parameters and will aim to make the first observation of asymmetries in neutrino and antineutrino oscillations arising from a CP-violating phase, shedding light on one of the most promising explanations for the matter-antimatter asymmetry in the Universe. The search for nucleon decays will probe one of the key tenets of Grand Unified Theories. In the case of a nearby supernova, Hyper-Kamiokande will observe an unprecedented number of neutrino events, providing much needed experimental results to researchers seeking to understand the mechanism of the explosion. Finally, the detection of astrophysical neutrinos from sources such as dark matter annihilation, gamma ray burst jets, and pulsar winds could further our understanding of some of the most spectacular, and least understood, phenomena in the Universe.

Hyper-Kamiokande employs a ring-imaging water Cherenkov detector technique and as a consequence, the capability of a water Cherenkov detector largely relies on the performance of its photosensors. The detector is segmented in two parts, an inner-detector surrounded by 4000020 " photomultipliers, and an outer-detector with 67008 " photomultipliers aimed to reject cosmic background. This setup is prompt to an optimization in both is inner and outer part, to achieve maximum sensitivity and reconstruction capability, and also taking in consideration costs and logistics.

The number of photons and their arrival times on the photomultipliers are used to reconstruct the energy and the vertex of the particle, respectively. New photodetectors are being developed for Hyper-Kamiokande, to achieve twice larger detection efficiency for Cherenkov photons, the superior photon counting and timing resolution compared to that used in Super-Kamiokande.

The detector will be located underground at Kamioka mine in Gifu Prefecture, with an overburden of $650 \mathrm{~m}$ or more of rock, which is equivalent to $1750 \mathrm{~m}$ or more of water. The target date for beginning the measurement with first tank is 2026. The measurement with the second tank will begin 6 years after that.

\section{Hyper-Kamiokande Detector}

The Hyper-Kamiokande experiment employs a ring-imaging water Cherenkov detector technique to detect rare interactions of neutrinos and the possible spontaneous decay of protons and bound neutrons. The detector water plays two roles: a target material for incoming neutrinos and source of nucleons to decay. The detector is filled with highly transparent purified water. A light attenuation length above $100 \mathrm{~m}$ can be achieved which allows us to detect a large fraction of the emitted Cherenkov light around the periphery of the water volume. An option being investigated is the Gd-doping of the water [1]. The neutrons captured on gadolinium produce an $8 \mathrm{MeV}$ gamma 


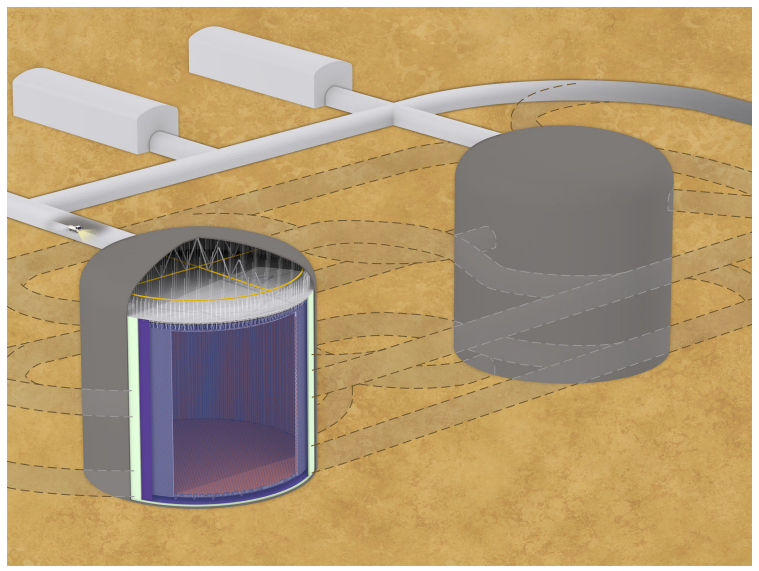

Figure 1: Schematic view of one Hyper-Kamiokande water Cherenkov detector [1]. Our proto-collaboration plans to construct two detector tanks consecutively, aiming the obsevations start at 2026 and 2032 respectively. Each tank will provide the fiducial volume of $0.187 \mathrm{Mt}$ ultra pure water, with the dimensions of the $74 \mathrm{~m}(\mathrm{D}) \times 60 \mathrm{~m}(\mathrm{H})$.

cascade after a few $\mu \mathrm{sec}$ from production by charge-current interaction between the neutrinos and water, providing a specific signature of the process for the analysis.

The detector is instrumented with an array of sensors with single-photon sensitivity in order to enable reconstruction of the spatial and timing distributions of the Cherenkov photons which are emitted by secondary particles from neutrino interactions and nucleon decays. The dimension of the photo-sensors and their density are subject to an optimization that takes into account the required signal identification efficiencies, background rejection power, and cost. HyperKamiokande's reconstruction performances is expected to meet or exceed that of its predecessor, Super-Kamiokande. Nominally the size and configuration of the two detectors are similar enough that event selections and systematic errors are not expected to differ largely.

The Hyper-Kamiokande detector is designed to employ newly developed high-efficiency and high-resolution PMTs (Hamamatsu R12860) which would amplify faint signatures such as neutron signatures associated with neutrino interactions, nuclear de-excitation gammas and $\pi^{+}$in proton decays into Kaons, and so on. This increased sensitivity greatly benefit the major goals of the Hyper-Kamiokande experiment such as clean proton decay searches and observation of supernova electron anti-neutrinos.

The photo-sensors have vacuum glass bulbs and will be located as much as $60 \mathrm{~m}$ underwater in the Hyper-Kamiokande cavern. At this depth, the applied pressure is close to the manufacturers upper specification of the Super-Kamiokande R3600 PMT (0.65 MPa).

The detector is instrumented with front-end electronics and a readout network/computer system. The system is capable of high-efficient data acquisition for two successive events in which Michel electron events follow muon events with a mean interval of $2 \mu \mathrm{sec}$. It is also able to collect the vast amount of neutrinos, which would come from nearby supernova in a nominal time period of $10 \mu \mathrm{sec}$. 


\section{Neutrino Oscillation}

\subsection{Introduction}

Neutrino oscillation was fomerly observed by the Super-Kamiokande experiment in 1998 [2] which implies that neutrinos have non-zero masses and flavor mixing, which was not predicted by the theory of the Standard Model of particle physics and therefore are the most tangible evidence of existing physics beyond this framework.

Even though the neutrino oscillation mechanism is fairly understood and most of this parameters are measured below $10 \%$ accuracy [3], which is already remarkable for particles interacting so little with matter, Hyper-Kamiokande would allow to improve the current bounds obtained by Super-Kamiokande on the still unknown parameters such as the CP phase - labelled as $\delta_{C P}-$ and the mass hierarchy, which is the sign of the largest square mass difference between neutrinos mass eigenstates, $\Delta m^{2} \equiv \Delta m_{31}^{2}=\Delta m_{32}^{2}$.

In this purpose the Hyper-Kamiokande experiment will use the $1300 \mathrm{~kW}$ beam (after a longterm upgrade) of $v_{\mu}$ produced by the J-PARC facility in Tokai, Japan, at $295 \mathrm{~km}$ from the detector. The beam will be 2.5 degrees off-axis from the Hyper-Kamiokande site, so the distribution of neutrinos arriving to the detector will be peaked at $600 \mathrm{MeV}$.

With a total exposure of $7.5 \mathrm{MW} \times 10^{7} \mathrm{sec}$ integrated proton beam power - equivalent to $1.56 \times 10^{22}$ protons on target with a $30 \mathrm{GeV}$ proton beam $-\delta_{C P}$ could be establish with an accuracy better than 21 degrees fo all possible values of $\delta_{C P}$ with more than $3 \sigma$ of statistical significance.

\subsection{Atmospheric neutrinos}

Neutrino oscillation parameters will be measured also using atmospherical sources, which can provide complimentary informations with respect to the long-baseline neutrino beam. This diversity of both energy and pathlength, which ranges from several order of magnitude starting at $\mathscr{O}(100) \mathrm{MeV}$ and between $\mathscr{O}(10)$ to $\mathscr{O}\left(10^{4}\right) \mathrm{km}$, makes atmospheric neutrinos a particularly versatile tool for studying neutrino oscillations.

That being said, the most apparent oscillation features are driven by the so-called atmospheric mixing parameters, $\theta_{23}$ and $\Delta m^{2}$ and they induce a deficit of observed upward-going $v_{\mu}$ interactions at predominantly multi-GeV energies as these neutrinos oscillates into primarly unobserved $v_{\tau}$. However, the value of $\theta_{13}$ is non-zero and the presence of matter effects will enhance the $v_{\mu} \rightarrow v_{e}$ for neutrinos ranged between [2-10] GeV, with probabilites up to $50 \%$ depending on the hierarchy - in this case, the normal one - while no enhancment will be seen in the antineutrinos channel (and it will be the opposite if the hierarchy is inverted).

Figure 2 shows the expected $v_{e}$ flux at the detector normalized to the unoscillated prediction under this approximation for four configuration of the oscillation parameters and neutrinos with zenith angle $\cos \theta_{v}=-0.8$. At energies between [5-10] $\mathrm{GeV}$ the most prominent feature of the figure is the parametric resonance driven by $\theta_{13}$ whose amplitude increases with $\sin ^{2} \theta_{23}$ (c.f. panels a. and b.). Further, this resonance becomes suppressed in the neutrino channel when the hierarchy is switched from normal to inverted (compare panels a. and d.).

Figure 3 shows the Hyper-Kamiokande's sensitivity to resolving the neutrino mass hierarchy (left panel) and the octant of $\theta_{23}$ assuming a single detectors after 10 years (right panel). Here the 

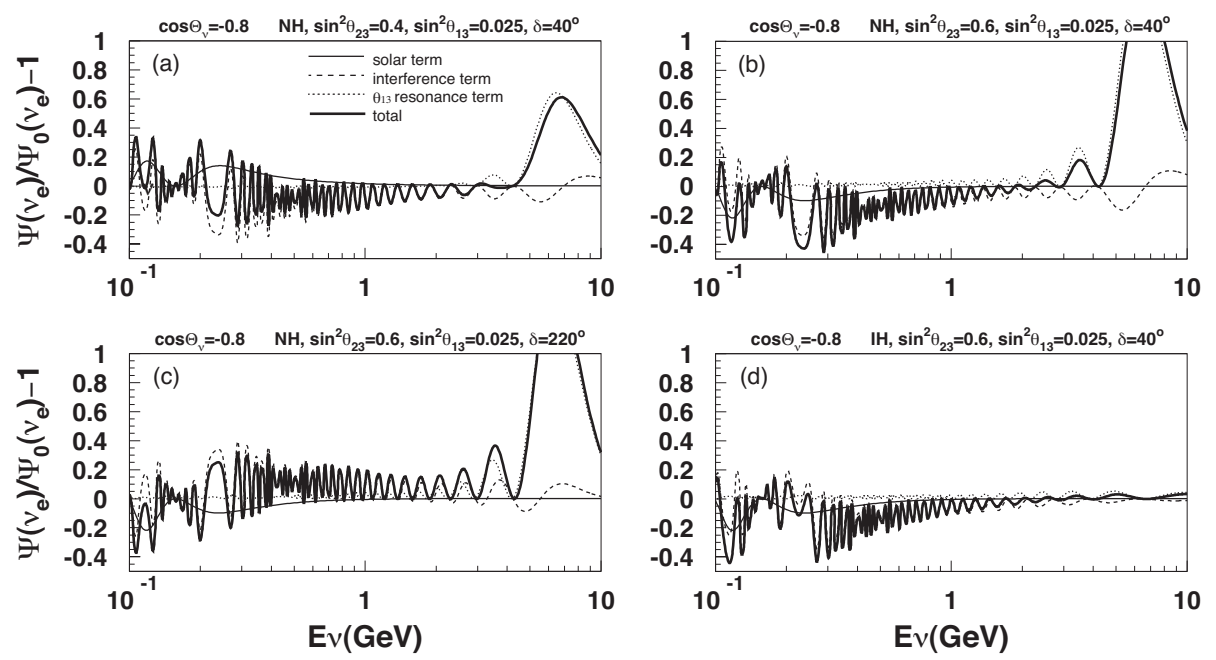

Figure 2: Oscillated $v_{e}$ flux relative to the non oscillated flux as a function of neutrino energy for the upward going neutrinos with zenith angle $\cos \theta_{v}=-0.8$ [1]. The different lines corresponds to the different terms inside the oscillation probability.

second detector is assumed to begin data taking 6 years after the start of the experiment. In each panel the sensitivity is defined as $\sqrt{\Delta \chi^{2}}$, which for mass hierarchy resolution corresponds to the difference $\chi^{2}$ between the actual real model and the alternate hypothesis.
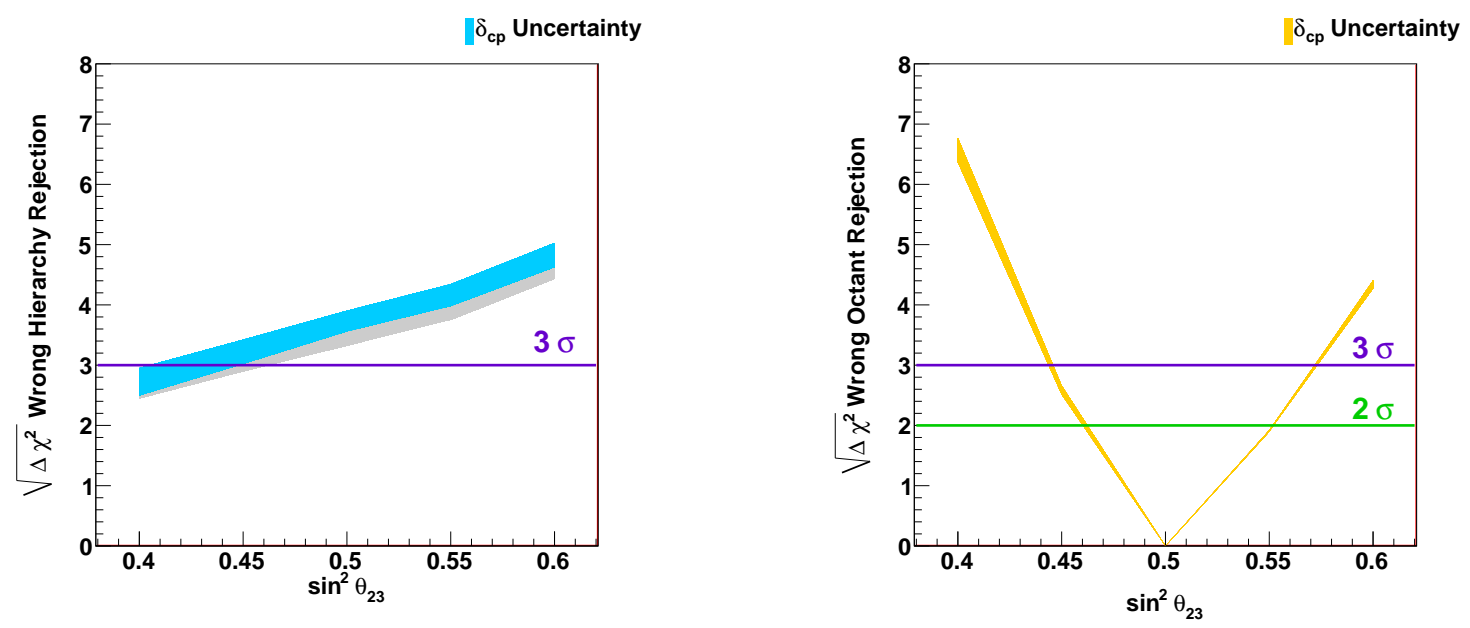

Figure 3: Neutrino mass hierarchy (left) and octant (right) sensitivity as function of the real value of $\sin ^{2} \theta_{23}$ after 10 years of data with one single tank. Uncertainty on $\delta_{C P}$ is shown by the width of the band.

Moreover, the Hyper-Kamiokande detector will be able to probe the existence of a light sterile neutrino state at $\Delta m_{s}^{2} \approx 1 \mathrm{eV}$, as hinted by a variety of short baseline experiment [4] [5]. Figure 4 shows the upper limit on the PMNS extended matric paremeter describing the sterile mixing. Other sub-dominant contributions to the standard oscillation picture, such as effect of Lorentz-invariance Violating (LV) process are expected to influence the oscillations of atmospheric neutrinos. Though Hyper-Kamiokande will have an atmospheric neutrino sample of unprecendented size, its increased 
sensitivity to sterile and LV oscillations relative to existing measurements is hampered by current understanding of atmospheric neutrinos flux and interactions uncertainties.
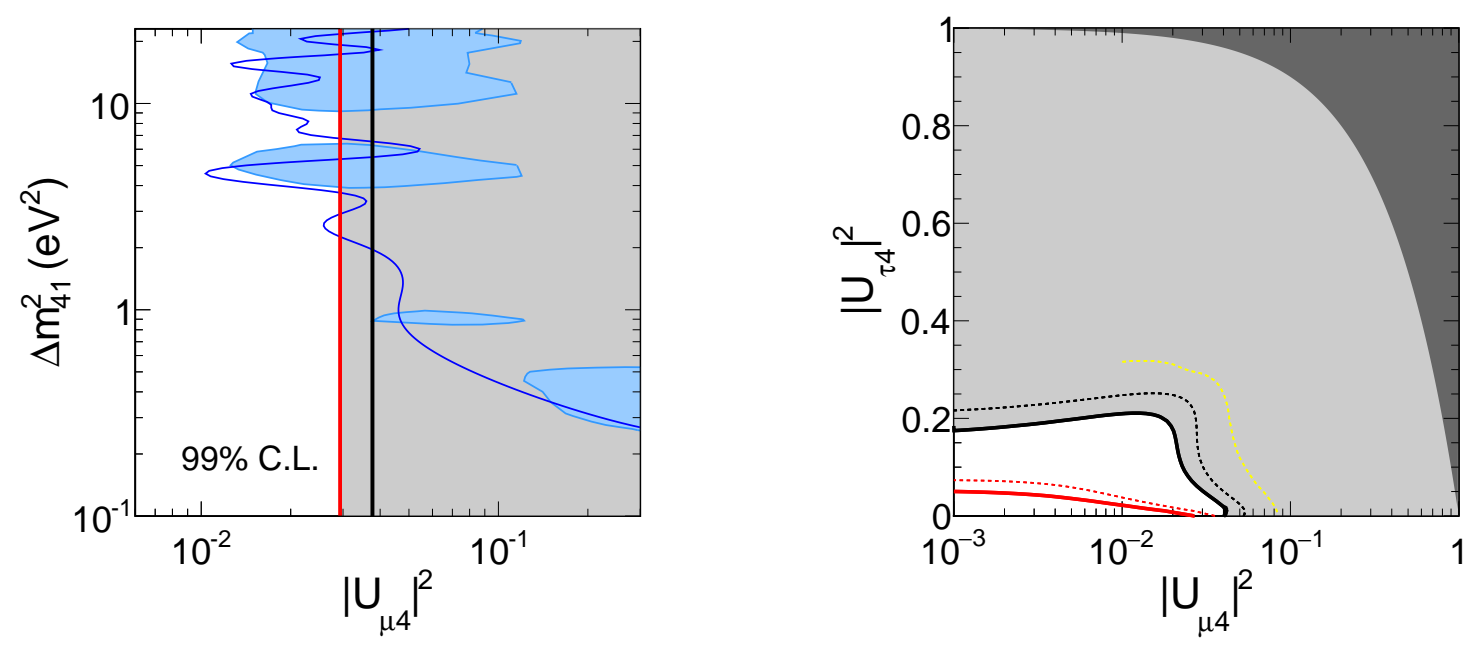

Figure 4: Expected 90\% C.L upper limit on $U_{\mu 4}^{2}$ (left) and $U_{\mu 4}^{2}$ vs $U_{\tau 4}^{2}$ (right) for 10 years of data taking (red) compared to the recent limits from Super-Kamiokande (black). $90 \%$ limits on the sterile parameters mixing extracted from the $v_{\mu}$ appearance channel in MiniBooNE and SciBooNE are drawn in blue and in light grey shows the allowed region from the $v_{e}$ joint fit of appearance and disappearance channels.

\subsection{Solar Neutrinos}

The Sun is an abundant and nearby source of neutrinos. Recently, Super-Kamiokande showed the first indication of the terrestrial matter effects on ${ }^{8} \mathrm{~B}$ solar neutrino oscillations. This was a direct confirmation of the MSW model predictions for neutrino interactions with matter, which is also used to describe neutrino behaviour as it travels through the Sun. Hyper-K hopes to measure terrestrial matter effects with higher precision to better understand neutrino oscillation behaviour in the presence of matter. This also might resolve the $2 \sigma$ tension between the current best fit values of $\delta m$ from solar and reactor neutrino experiments (see Figure 5), which is thought to be due to solar neutrino interactions in matter. Additionally, there are several physics goals for the solar neutrino observations in Hyper-Kamiokande, such as long and short time variation of the ${ }^{8} \mathrm{~B}$ flux, the first measurement of hep neutrinos, and precise measurement of solar neutrino energy spectrum.

\section{Neutrino Astrophysics}

\subsection{Supernova}

Core collapse supernova explosion releases almost 99\% of its energy into neutrinos, in which all three types of neutrinos and antineutrinos are emitted. In 1987, the former Kamiokande, IMB and Baksan experiments only measured a handful (25) neutrinos coming from the supernova burst of SN1987a, but mark their discovery as the invention of neutrino astronomy observations.

If a supernova explosion occurs halfway across our galaxy, the Hyper-Kamiokande detector would detect approximately 52000 to 79000 neutrino events per tank. Hyper-Kamiokande can also 


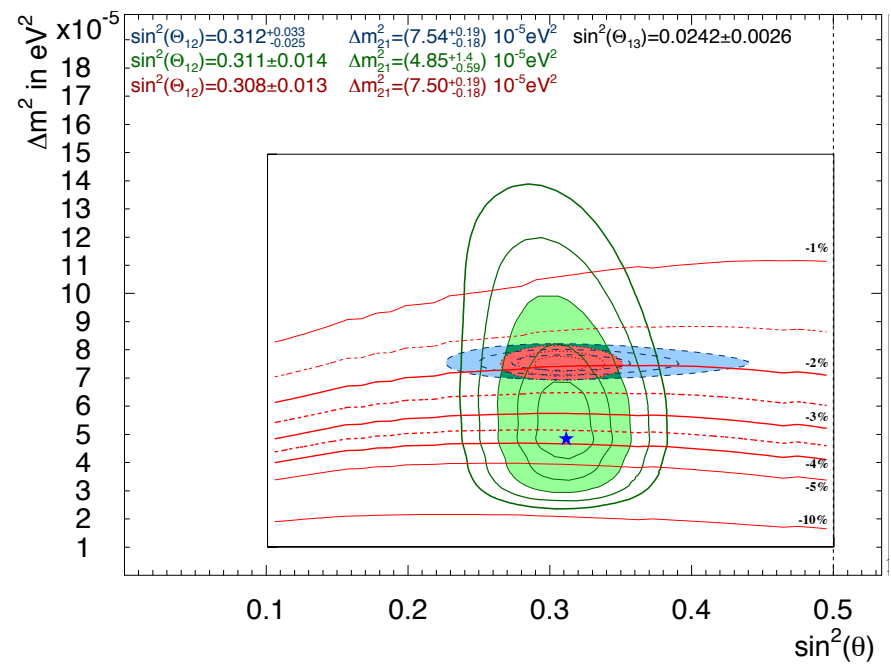

Figure 5: Allowed neutrino oscillation parameter region from all the solar neutrino experiments (green), reactor neutrino from KamLAND (blue) and combined (red) from one to ve sigma lines and three sigma filled area. The star shows the best fit parameter from the solar neutrinos.

provide information for neutrinos with energy down to $\approx 3 \mathrm{MeV}$ and determine their direction. Such a low threshold can be use during analysis of supernovas events because supernovas burst neutrinos are localized in a short time period.

Compared with the current experiment or planned experiment such as Super-Kamiokande ; ice Cherenkov detectors IceCube/PINGU, or the Liquid Argon (LAr) TPC DUNE experiment, HyperKamiokande will have 8 to 16 times larger fiducial volumes than Super-Kamiokande for example, and an order of magnitude more compared to kT liquid argon DUNE. Moreover, LArTPCs' lack of free protons means that the inverse- $\beta$ decay reaction won't be observed. The low energy threshold of $\approx 3 \mathrm{MeV}$ is also much lower than neutrino telescope such as IceCube/PINGU (about $15 \mathrm{MeV}$ ). With these advantages, the measurement with Hyper-Kamiokande should at least reveal the detail mechanism of supernova explosions [1].

\subsection{Dark Matter searches}

Hyper-Kamiokande will be extremely sensitive to the energy range of neutrinos from annihilations of light (below $100 \mathrm{GeV}$ ) WIMP dark matter, a region which is suggested by recent direct dark matter search experiments. Hyper-Kamiokande can search for dark matter WIMPs by looking for neutrinos created in pair annihilation from trapped dark matter in the Galactic centre or the centre of the Sun. Atmospheric neutrinos are a background to this WIMP search, so spacial cuts are made to determine if there is an excess of neutrinos coming from the Galactic centre or the Sun. Hyper-Kamiokande will have the ability to detect both $v_{e}$ and $v_{\mu}$ components of the signal, making it more sensitive to this type of analysis. Figure 6 shows the spin indepedant WIMPs nucleon scattering cross section in function of WIMPs mass search in different channel, and the sensitivity of the Hyper-Kamiokande detector. 


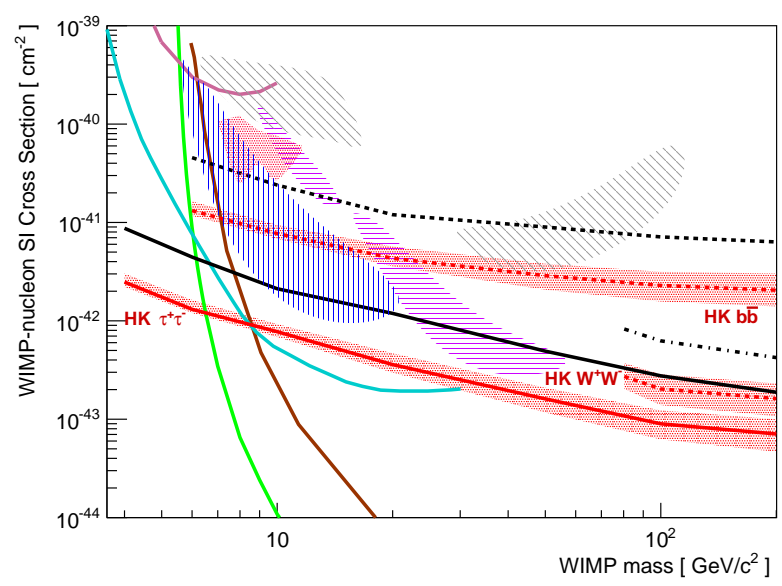

Figure 6: $90 \%$ C.L upper limits on the spin indepedant WIMP nucleon scattering cross section based on a search from WIMP induced neutrinos coming from the center of the earth for several annihilation channels. Limits (lines) and allowed region (filled area) from other experiments and sensitivity for Hyper-Kamiokande are represented.

\section{Summary}

The Hyper-Kamiokande experiment as a rich physics program which depends on the performances of the cylindrical water Cherenkov detector, for energy and vertex reconstruction. The sensitivity of the detector allows to study atmospheric and astrophysical neutrinos, down to $\approx 3 \mathrm{MeV}$ events produced by supernovas burst neutrinos for example, and in complimentarity with next generation experiment such as DUNE or KM3NeT. Furthermore, the detector will allow in 10 years data taking to adress the questions of $\delta_{C P}$ and the neutrino mass hierarchy, in addition to research for more beyond Standard Model physics, such as WIMPs or sterile neutrinos signal search.

\section{References}

[1] K. Abe et al., Letter of Intent: The Hyper-Kamiokande Experiment — Detector Design and Physics Potential.

[2] Y. Fukuda et al. (Super-Kamiokande), Phys. Rev. Lett. 81 , 1562 (1998), arXiv:hep-ex/9807.003.

[3] K. Abe et al. (Super-Kamiokande), Phys. Rev. D83 , 052010 (2011), arXiv:hep-ex/1010.0118. Y. Ashie et al. (Super-Kamiokande), Phys. Rev. D71 , 112005 (2005), arXiv:hep-ex/0501.064. Y. Ashie et al. (Super-Kamiokande), Phys. Rev. Lett. 93 , 101801 (2004), arXiv:hep-ex/0404.034.

[4] A. Aguilar-Arevalo et al. (LSND), Phys. Rev. D64 , 112007 (2001), arXiv:hep-ex/0104049. A. Aguilar-Arevalo et al. (MiniBooNE), Phys.Rev.Lett. 110 , 161801 (2013), arXiv:hep-ex/1207.4809.

[5] P. Huber, Phys.Rev. C84 , 024617 (2011), arXiv:hep-ex/1106.0687. G. Mention et al. , Phys.Rev. D83 , 073006 (2011), arXiv:hep-ex/1101.2755. 